\title{
A post-marketing observational study of ramucirumab in patients with gastric cancer in Japan
}

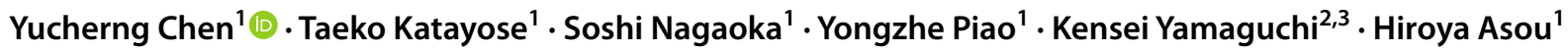

Received: 24 November 2020 / Accepted: 16 May 2021 / Published online: 28 May 2021

(c) The Author(s) 2021

\begin{abstract}
Background This study evaluated the safety and effectiveness of ramucirumab monotherapy and combination therapy for advanced gastric cancer in the real-world setting.

Methods This single-arm, prospective, multicenter, non-interventional, observational, post-marketing study was conducted in Japan from August 2015 to March 2019. Patients with unresectable advanced or recurrent gastric cancer and newly prescribed ramucirumab were followed for up to 12 months after first treatment. Data on adverse events and survival were collected via Electronic Data Capture.

Results Of 687 enrolled patients, 658 were eligible for analysis. Most patients received either ramucirumab monotherapy $(123 / 658 ; 18.7 \%)$ or ramucirumab plus paclitaxel combination therapy $(528 / 658 ; 80.2 \%)$. The majority of patients reported $\geq 1$ adverse events in both the combination therapy (any grade, $479 / 528 ; 90.7 \% ; \geq$ Grade $3,321 / 528 ; 60.8 \%$ ) and monotherapy groups (any grade, $77 / 123 ; 62.6 \% ; \geq$ Grade $3,42 / 123 ; 34.2 \%$ ). The most common any grade adverse events were neutropenia (combination: 49.6\%; monotherapy: 8.9\%), fatigue (combination: $19.5 \%$; monotherapy: $13.8 \%$ ), and decreased appetite (combination: 18.2\%; monotherapy: 10.6\%). Grade 5 adverse events were reported in 4 patients, including metastases to meninges, pneumonia aspiration, death, and gastric perforation; of these, gastric perforation was deemed treatment-related. Median survival time was 5.7 months (95\% confidence interval: 4.1-6.8 months) following monotherapy and 11.0 months (95\% confidence interval: 9.8-12.2 months) following combination therapy.

Conclusions This analysis adds to the limited data available on ramucirumab use in a real-world setting, demonstrating similar safety and effectiveness for ramucirumab in treating advanced gastric cancer in routine clinical practice in Japan to that of global clinical trials.
\end{abstract}

Keywords Ramucirumab $\cdot$ Post-marketing surveillance $\cdot$ Gastric cancer

\section{Introduction}

Gastric cancer is the fifth most commonly diagnosed and third most deadly cancer globally [1]. Incidence rates are higher in Asian countries, including Japan, where gastric cancer is the second most common cancer [2]. Combination therapies with platinum and fluoropyrimidine agents are

Yucherng Chen

chen_yucherng@lilly.com

1 Eli Lilly Japan K.K., Lilly Plaza One Building, 5-1-28 Isogamidori, Chuo-Ku, Kobe, Hyogo 651-0086, Japan

2 Department of Gastroenterological Chemotherapy, Cancer Institute Hospital of Japanese Foundation for Cancer Research, Tokyo, Japan

3 Saitama Cancer Center, Saitama, Japan currently the standard first-line treatment for unresectable advanced gastric cancer [3]; however, survival is poor and second-line treatment options are limited.

In global phase 3 studies of gastric cancer, ramucirumab, a human IgG1 monoclonal antibody against vascular endothelial growth factor receptor-2 [4], has demonstrated significant survival benefits and an acceptable safety profile in the second-line setting. In patients with advanced gastric cancer who had progressed on platinum/fluoropyrimidinebased therapies, progression-free survival and overall survival were significantly improved with ramucirumab, both as a monotherapy and in combination with paclitaxel (ramucirumab + paclitaxel) [5, 6]. Globally, ramucirumab + paclitaxel is currently a preferred second-line treatment option for patients with advanced gastric cancer who have progressed after first-line chemotherapy $[3,7,8]$ and is the only 
recommended 'evidence level A' second-line regimen in the current Japanese gastric cancer treatment guidelines [9]. However, the majority of data on ramucirumab treatment is from clinical trials, and real-world data on ramucirumab treatment in gastric cancer patients are limited [10-13].

The objective of this study was to evaluate the safety and effectiveness of ramucirumab treatment in patients with unresectable advanced or recurrent gastric cancer in routine clinical practice in Japan. Data for ramucirumab monotherapy and ramucirumab combination therapy are described separately. Given the widespread use of ramucirumab for the treatment of advanced gastric cancer, we anticipate that these data will be referential and informative not only for clinicians in Japan but also for healthcare providers worldwide.

\section{Materials and methods}

\section{Patients}

This study includes patients with unresectable advanced/ recurrent gastric cancer who were being treated for the first time with ramucirumab under routine clinical practice in Japan. This study had no exclusion criteria. Patients included in this analysis received $\geq 1$ dose of ramucirumab. The study was conducted in compliance with the Good Post-marketing Study Practice in Japan.

\section{Study design and data collection}

This was a single-arm, prospective, multicenter, non-interventional, observational, post-marketing study conducted from August 17, 2015 (first-patient visit) to March 18, 2019 (database lock). Data were reported by investigators at participating medical facilities via a case report form (CRF) in an Electronic Data Capture system. Patients were observed for up to 12 months following the first dose of ramucirumab. For safety data, follow-up observation was carried out for 30 days after discontinuation of ramucirumab or post-discontinuation treatment was started, whichever was earlier. For survival data, follow-up continued past 12 months, if allowed by investigators.

All treatment decisions were at the investigator's discretion. The approved adult dosage for gastric cancer in Japan is $8 \mathrm{mg} / \mathrm{kg}$ (body weight) of ramucirumab, administered intravenously over 60 min every 2 weeks, with adjustments by the investigator allowed as deemed necessary for the patient's condition.

Data were collected on patient demographic and clinical characteristics, treatment history, concomitant therapies, safety, and survival. Adverse events (AEs), serious adverse events (SAEs), and adverse events of special interest (AESIs) were classified using Medical Dictionary for
Regulatory Activities, Version 21.1, preferred terms (consolidated terms where indicated) and graded based on the National Cancer Institute Common Terminology Criteria for Adverse Events, Version 4.0. SAEs were defined according to the International Council for Harmonisation of Technical Requirements for Pharmaceuticals for Human Use guideline E2A. Survival status was defined from the date of first ramucirumab treatment to the date of death (any cause) or until the last day of observation, as confirmed in the CRF.

\section{Statistical analysis}

Continuous variables were summarized using mean (standard deviation [SD]) and median. Categorical variables were summarized using frequency and incidence. If baseline data were missing, the patient was counted as 'not described' or 'not measured' in the relevant categories. Data were analyzed for the overall study population and by treatment, categorized as ramucirumab monotherapy, ramucirumab + paclitaxel therapy, other ramucirumab combination therapy, and unspecified ramucirumab therapy (monotherapy/combination therapy details not provided by investigator). Patients were categorized in the ramucirumab + paclitaxel group if paclitaxel was administered on the same day as ramucirumab $\geq 1$ time during the study period and in the other ramucirumab combination therapy group if another drug(s) (but no paclitaxel) was administered concomitantly with ramucirumab. Data for the other ramucirumab combination and unspecified ramucirumab therapy groups are included in the overall analysis population and are not separately summarized due to small sample size. Subgroup analyses examined treatment outcomes relative to selected baseline characteristic categories to determine factors affecting ramucirumab safety and effectiveness. Analyses were exploratory in nature. For comparisons between treatment groups, no covariate adjustments were made and $p$ values are not reported. Survival status was estimated using the Kaplan-Meier method [14], including median survival time and associated $95 \%$ confidence intervals (CIs).

\section{Results}

\section{Patients and treatments}

In total, 687 patients were enrolled in the study. CRFs were collected for 683 patients (Online Resource 1). Of these, 25 patients were excluded, the majority of whom $(n=16 ; 64 \%)$ because they did not receive ramucirumab. A total of 658 patients were included in both the safety and the effectiveness analysis datasets, of which $123(18.7 \%)$ received ramucirumab monotherapy, $532(80.9 \%)$ received ramucirumab in combination with another anti-cancer drug, and $3(0.5 \%)$ 
received unspecified ramucirumab treatment. Paclitaxel was the most common anti-cancer drug used concomitantly (528/532; 99.2\%) whereas four patients $(0.8 \%)$ received ramucirumab in combination with another anti-cancer drug ( $\geq 1$ of irinotecan hydrochloride hydrate, fluorouracil, docetaxel, and/or cisplatin).

\section{Demographics and baseline characteristics}

The majority of patients were male $(n=463 ; 70.4 \%)$ with a median age of 68.0 years (Table 1 ). Patients were diagnosed with metastatic $(n=652 ; 99.1 \%)$ cancer of the stomach $(n=609 ; 92.6 \%)$ or gastroesophageal junction $(n=48$; $7.3 \%$ ), with a baseline Eastern Cooperative Oncology Group Performance Status (ECOG PS) of $0(n=321 ; 48.8 \%)$ or 1 $(n=272 ; 41.3 \%)$. The majority of patients $(n=436 ; 66.3 \%)$ received ramucirumab as a second-line treatment, with $11.9 \%(n=78)$ receiving ramucirumab as first-line treatment and $21.7 \%(n=143)$ receiving ramucirumab as thirdline or later treatment. Ramucirumab was received as lateline ( $\geq 3 \mathrm{rd}$ ) therapy in $46.3 \%$ of patients in the monotherapy group and $15.5 \%$ patients in the ramucirumab + paclitaxel group. ECOG PS was $\geq 2$ in $24.4 \%$ and $5.9 \%$ of patients in the monotherapy and ramucirumab + paclitaxel groups, respectively. Overall, 98.8\% $(n=650)$ of patients had received prior systemic chemotherapy, including adjuvant therapy, for gastric cancer, (Online Resource 2), most commonly tegafur/gimeracil/oteracil potassium combination drug $(n=542 ; 83.4 \%)$, cisplatin $(n=282 ; 43.4 \%)$, and oxaliplatin $(n=268 ; 41.2 \%)$.

Mean (SD) duration of therapy was 19.9 (15.7) weeks (median: 16.0 weeks) for ramucirumab (Table 2). Overall, the mean (SD) dose intensity for ramucirumab was 3.4 (0.6) $\mathrm{mg} / \mathrm{kg} /$ weeks (median $3.6 \mathrm{mg} / \mathrm{kg} /$ week) and mean (SD) relative dose intensity was $85.1 \%$ (16.0) (median: $89.3 \%$ ). The mean duration of therapy (SD) was 11.1 (12.6) weeks in the monotherapy group and 21.9 (15.6) weeks in the ramucirumab + paclitaxel group. The mean (SD) relative dose intensity was $92.2 \%$ (13.1) in the monotherapy group and $83.5 \%$ (16.2) in the ramucirumab + paclitaxel group (Table 2).

\section{Safety}

Overall, AEs were observed in 561 (85.3\%) patients, and $\geq$ Grade 3 AEs were observed in 365 patients (55.5\%). Neutropenia $(n=273 ; 41.5 \%)$, fatigue $(n=124,18.8 \%)$, decreased appetite $(n=112 ; 17.0 \%)$, and hypertension $(n=103 ; 15.7 \%)$ were the most common AEs of any grade whereas neutropenia $(n=208 ; 31.6 \%)$, leukopenia $(n=44 ; 6.7 \%)$, and febrile neutropenia $(n=39 ; 5.9 \%)$ were the most common $\geq$ Grade 3 AEs. The majority of patients reported $\geq 1 \mathrm{AE}$ (monotherapy: any grade, 77/123;
$62.6 \%$; $\geq$ Grade 3, 42/123; 34.2\%; ramucirumab + paclitaxel: any grade, $479 / 528 ; 90.7 \% ; \geq$ Grade $3,321 / 528 ; 60.8 \%$; Fig. 1). AEs commonly known to occur with paclitaxel were reported in the ramucirumab + paclitaxel group (e.g., neutropenia: any grade, $49.6 \%$; $\geq$ Grade $3,38.3 \%$; leukopenia: any grade, $14.4 \% ; \geq$ Grade $3,8.1 \%$; peripheral neuropathy: any grade, $14.0 \%$; Grade $3,1.1 \%$; alopecia: any grade, $14.6 \% ; \geq$ Grade, $30.2 \%$ ) and were numerically less frequent in the monotherapy group (neutropenia: any grade, $8.9 \% ; \geq$ Grade $3,4.9 \%$; leukopenia: any grade, $2.5 \% ; \geq$ Grade $3,0.8 \%$; peripheral neuropathy: any grade, $4.1 \%$; $\geq$ Grade 3, 0.8\%; alopecia: any grade, $0.8 \% ; \geq$ Grade 3, $0 \%$; Fig. 1). The majority of common AEs $(\geq 70 \%)$ were reported as resolved or resolving by data cutoff at the end of the study period (Online Resource 3). Overall, the incidence rates of interstitial lung disease (ILD) and pneumonitis were $0.9 \%$ (monotherapy, $n=1$; ramucirumab + paclitaxel, $n=5$ ) and $0.8 \%$ (ramucirumab + paclitaxel, $n=5$ ), respectively, all of which were $\leq$ Grade 3 .

SAEs were reported in 153 (23.3\%) patients (monotherapy: 23/123; 18.7\%; ramucirumab + paclitaxel: 129/528; $24.4 \%)$. The most commonly reported SAEs in both the ramucirumab monotherapy and ramucirumab + paclitaxel groups were neutropenia (monotherapy: $n=3 ; 2.4 \%$; ramucirumab + paclitaxel: $n=35 ; 6.6 \%$ ), febrile neutropenia (monotherapy: $n=2 ; 1.6 \%$; ramucirumab + paclitaxel: $n=24 ; 4.6 \%$ ), and decreased appetite (monotherapy: $n=2$; 1.6\%; ramucirumab + paclitaxel: $n=12 ; 2.3 \%$; Fig. 1$)$. The incidence of SAEs of ILD and pneumonitis was $0.6 \%(n=3)$ and $0.2 \%(n=1)$, respectively, in the ramucirumab + paclitaxel group and $0.8 \%(n=1)$ for ILD in the monotherapy group. Grade 5 AEs were reported for 4 patients ( $n=1$ each), including metastases to meninges and gastric perforation in the monotherapy group and pneumonia aspiration and death in the ramucirumab + paclitaxel group. Gastric perforation was the only grade $5 \mathrm{AE}$ deemed related to ramucirumab. The patient experienced gastric perforation at the location of the primary tumor 2 weeks after starting ramucirumab, and the cause of death was reported as disease progression with bleeding from the perforation site.

AESIs for ramucirumab are summarized by treatment in Online Resource 4. Overall, patients most commonly experienced AESIs of hypertension (any grade $n=103$; $15.7 \%$; $\geq$ Grade $3 n=37,5.6 \%$ ), bleeding/hemorrhage events (any grade $n=73,11.1 \% ; \geq$ Grade $3 n=13,2 \%$ ), including gastrointestinal hemorrhage events (any grade $n=17$, $2.6 \%$; Grade $3 n=9,1.4 \%$ ), proteinuria (any grade $n=60$; $9.1 \%$; $\geq$ Grade $3 n=20,3.0 \%$ ), and liver injury/liver failure (any grade $n=39 ; 5.9 \% ; \geq$ Grade $3 n=9,1.4 \%$ ).

The incidence of AEs by baseline clinical characteristics is shown in Online Resource 5. The incidence of AEs was not found to differ substantially by primary tumor site, metastatic or recurrent tumor site, or advanced age $(\geq 75)$. 
Table 1 Patient demographics and baseline clinical characteristics

\begin{tabular}{|c|c|c|c|}
\hline Characteristic, $n(\%)$ & $\begin{array}{l}\text { Ramucirumab monotherapy } \\
N=123\end{array}$ & $\begin{array}{l}\text { Ramucirumab + paclitaxel } \\
N=528\end{array}$ & $\begin{array}{l}\text { Overall analy- } \\
\text { sis population } \\
N=658^{\mathrm{a}}\end{array}$ \\
\hline \multicolumn{4}{|l|}{ Sex } \\
\hline Male & 84 (68.3) & 375 (71.0) & $463(70.4)$ \\
\hline Female & $39(31.7)$ & $153(29.0)$ & $195(29.6)$ \\
\hline \multicolumn{4}{|l|}{ Age, years } \\
\hline Median & 69.0 & 68.0 & 68.0 \\
\hline Minimum-maximum & $21-88$ & $29-94$ & $21-94$ \\
\hline$\geq 75$ years & $39(31.7)$ & $107(20.3)$ & $147(22.3)$ \\
\hline \multicolumn{4}{|l|}{ Body Mass Index, $\mathrm{kg} / \mathrm{m}^{2}$} \\
\hline Median & 19.3 & 19.8 & 19.8 \\
\hline Minimum-maximum & $12.3-28.3$ & $12.9-32.9$ & $12.3-32.9$ \\
\hline \multicolumn{4}{|l|}{ HER2 status } \\
\hline Positive & $26(21.1)$ & $102(19.3)$ & $128(19.5)$ \\
\hline Negative & $87(70.7)$ & $380(72.0)$ & 473 (71.9) \\
\hline Unknown or not done & $10(8.1)$ & $46(8.7)$ & $56(8.5)$ \\
\hline Not described & $0(0.0)$ & $0(0.0)$ & $1(0.2)$ \\
\hline \multicolumn{4}{|l|}{ Primary tumor site } \\
\hline Gastric & $116(94.3)$ & $488(92.4)$ & $609(92.6)$ \\
\hline Gastroesophageal junction & $7(5.7)$ & $40(7.6)$ & $48(7.3)$ \\
\hline Not described & $0(0.0)$ & $0(0.0)$ & $1(0.2)$ \\
\hline \multicolumn{4}{|l|}{ Presence of residual primary tumor } \\
\hline No & $61(49.6)$ & $252(47.7)$ & $316(48.0)$ \\
\hline Yes & $62(50.4)$ & $276(52.3)$ & $341(51.8)$ \\
\hline Not described & $0(0.0)$ & $0(0.0)$ & $1(0.2)$ \\
\hline \multicolumn{4}{|l|}{ Metastasis and recurrent sites } \\
\hline No & $0(0.0)$ & $5(1.0)$ & $5(0.8)$ \\
\hline Yes & $123(100)$ & $523(99.1)$ & $652(99.1)$ \\
\hline Peritoneal seeding (with ascites) & $42(34.2)$ & $158(30.2)$ & $201(30.8)$ \\
\hline Peritoneal seeding (no ascites) & $19(15.5)$ & $123(23.5)$ & $143(21.9)$ \\
\hline Lymph nodes (intraperitoneal) & $62(50.4)$ & $273(52.2)$ & $337(51.7)$ \\
\hline Lymph nodes (other) & $21(17.1)$ & $61(11.7)$ & $83(12.7)$ \\
\hline Liver & $44(35.8)$ & $148(28.3)$ & $194(29.8)$ \\
\hline Lung & $25(20.3)$ & $48(9.2)$ & $73(11.2)$ \\
\hline Bone & $6(4.9)$ & $29(5.5)$ & $35(5.4)$ \\
\hline Brain & $1(0.8)$ & $2(0.4)$ & $3(0.5)$ \\
\hline Other & $13(10.6)$ & $63(12.1)$ & $76(11.7)$ \\
\hline Not described & $0(0.0)$ & $0(0.0)$ & $1(0.2)$ \\
\hline \multicolumn{4}{|l|}{ Treatment line (ramucirumab) ${ }^{b}$} \\
\hline $1 \mathrm{st}$ & $4(3.3)$ & $74(14.0)$ & $78(11.9)$ \\
\hline 2nd & $62(50.4)$ & $372(70.5)$ & $436(66.3)$ \\
\hline 3rd & $20(16.3)$ & $63(11.9)$ & $87(13.2)$ \\
\hline$\geq 4$ th & $37(30.1)$ & $19(3.6)$ & $56(8.5)$ \\
\hline Not described & $0(0.0)$ & $0(0.0)$ & $1(0.2)$ \\
\hline \multicolumn{4}{|c|}{ Eastern cooperative oncology group Performance status } \\
\hline 0 & $38(30.9)$ & $281(53.2)$ & $321(48.8)$ \\
\hline 1 & $53(43.1)$ & $215(40.7)$ & $272(41.3)$ \\
\hline 2 & $26(21.1)$ & $28(5.3)$ & $54(8.2)$ \\
\hline$\geq 3$ & $4(3.3)$ & $3(0.6)$ & $7(1.1)$ \\
\hline Not done & $2(1.6)$ & $1(0.2)$ & $4(0.6)$ \\
\hline
\end{tabular}


Table 1 (continued)

\begin{tabular}{llll}
\hline Characteristic, $n(\%)$ & $\begin{array}{l}\text { Ramucirumab monotherapy } \\
N=123\end{array}$ & $\begin{array}{l}\text { Ramucirumab+paclitaxel } \\
N=528\end{array}$ & $\begin{array}{l}\text { Overall analy- } \\
\text { sis population } \\
N=658^{\mathrm{a}}\end{array}$ \\
\hline Complications & & & \\
No & $43(35.0)$ & $216(40.9)$ & $262(39.8)$ \\
Yes & $80(65.0)$ & $312(59.1)$ & $395(60.0)$ \\
High blood pressure & $33(41.3)$ & $145(46.5)$ & $181(45.8)$ \\
Renal disease & $10(12.5)$ & $60(19.2)$ & $70(17.7)$ \\
Liver disease & $6(7.5)$ & $26(8.3)$ & $32(8.1)$ \\
Thromboembolism & $3(3.8)$ & $22(7.1)$ & $25(6.3)$ \\
Inflammatory digestive tract disease & $0(0.0)$ & $3(1.0)$ & $3(0.8)$ \\
Hemorrhagic diathesis and coagulation disorder & $0(0.0)$ & $2(0.6)$ & $2(0.5)$ \\
Wound healing disorder after major surgery & $0(0.0)$ & $1(0.3)$ & $1(0.3)$ \\
Other & $61(76.3)$ & $208(66.7)$ & $270(68.4)$ \\
Not described & $0(0.0)$ & $0(0.0)$ & $1(0.2)$ \\
\hline
\end{tabular}

HER2 human epidermal growth factor receptor-2, $N$ number of patients in population, $n$ number of patients in category

${ }^{a}$ Includes two subgroups with data not shown separately due to small sample size: patients who received other ramucirumab combination therapies $(N=4)$ or unspecified ramucirumab therapy $(N=3)$

${ }^{\mathrm{b}}$ Treatment line as reported by the investigator

Table 2 Dose duration and intensity of ramucirumab and paclitaxel

\begin{tabular}{|c|c|c|c|c|}
\hline & \multirow{2}{*}{$\begin{array}{l}\text { Ramucirumab mon- } \\
\text { otherapy } N=123 \\
\text { Ramucirumab }\end{array}$} & \multicolumn{2}{|c|}{$\begin{array}{l}\text { Ramucirumab + paclitaxel } \\
N=528\end{array}$} & \multirow{2}{*}{$\begin{array}{l}\text { Overall analy- } \\
\text { sis population } \\
N=658^{\mathrm{a}} \\
\text { Ramucirumab }\end{array}$} \\
\hline & & Ramucirumab & Paclitaxel & \\
\hline \multicolumn{5}{|c|}{ Duration of therapy, weeks } \\
\hline$n$ & 123 & 528 & 528 & 658 \\
\hline Mean (SD) & $11.1(12.6)$ & $21.9(15.6)$ & $21.4(15.3)$ & $19.9(15.7)$ \\
\hline Median & 6.3 & 18.0 & 17.9 & 16.0 \\
\hline Minimum-maximum & $2.0-53.0$ & $2.0-72.3$ & $2.0-76.0$ & $2.0-72.3$ \\
\hline \multicolumn{5}{|c|}{ Dose intensity ${ }^{\mathrm{b}}, \mathrm{mg} / \mathrm{kg} /$ week } \\
\hline$n$ & 123 & $527^{\mathrm{c}}$ & $518^{\mathrm{d}}$ & 656 \\
\hline Mean (SD) & $3.7(0.5)$ & $3.3(0.6)$ & $40.1(11.9)$ & $3.4(0.6)$ \\
\hline Median & 3.9 & 3.5 & 40.0 & 3.6 \\
\hline Minimum-maximum & $1.8-4.4$ & $1.1-4.7$ & $6.8-96.7$ & $1.1-4.7$ \\
\hline \multicolumn{5}{|c|}{ Relative dose intensity ${ }^{\mathrm{b}}, \%$} \\
\hline$n$ & 123 & $527^{\mathrm{c}}$ & $518^{\mathrm{d}}$ & 656 \\
\hline Mean (SD) & $92.2(13.1)$ & $83.5(16.2)$ & $66.8(19.8)$ & $85.1(16.0)$ \\
\hline Median & 97.8 & 86.9 & 66.7 & 89.3 \\
\hline Minimum-maximum & $44.7-109.1$ & $26.2-118.5$ & $11.3-161.1$ & $26.2-118.5$ \\
\hline
\end{tabular}

$N$ number of patients in population, $n$ number of patients in category, $S D$ standard deviation

${ }^{a}$ Includes two subgroups with data not shown separately due to small sample size: patients who received other ramucirumab combination therapies $(N=4)$ or unspecified ramucirumab therapy $(N=3)$

${ }^{b}$ Dose intensity refers to the actual amount of drug administered per week, calculated per patient as the total dose of ramucirumab $(\mathrm{mg})$ per body weight $(\mathrm{kg})$ per week of treatment. Relative dose intensity refers to the percentage of drug administered relative to the planned dose intensity

${ }^{c}$ One patient not included, as weight not provided

${ }^{\mathrm{d}}$ Nine patients not included, as dose information provided was insufficient for dose intensity calculations
As oxaliplatin is associated with acute and cumulative neurotoxicity [15], we also compared AE incidence of patients with and without prior oxaliplatin treatment. Over the study period, total AE incidence was similar between the two 


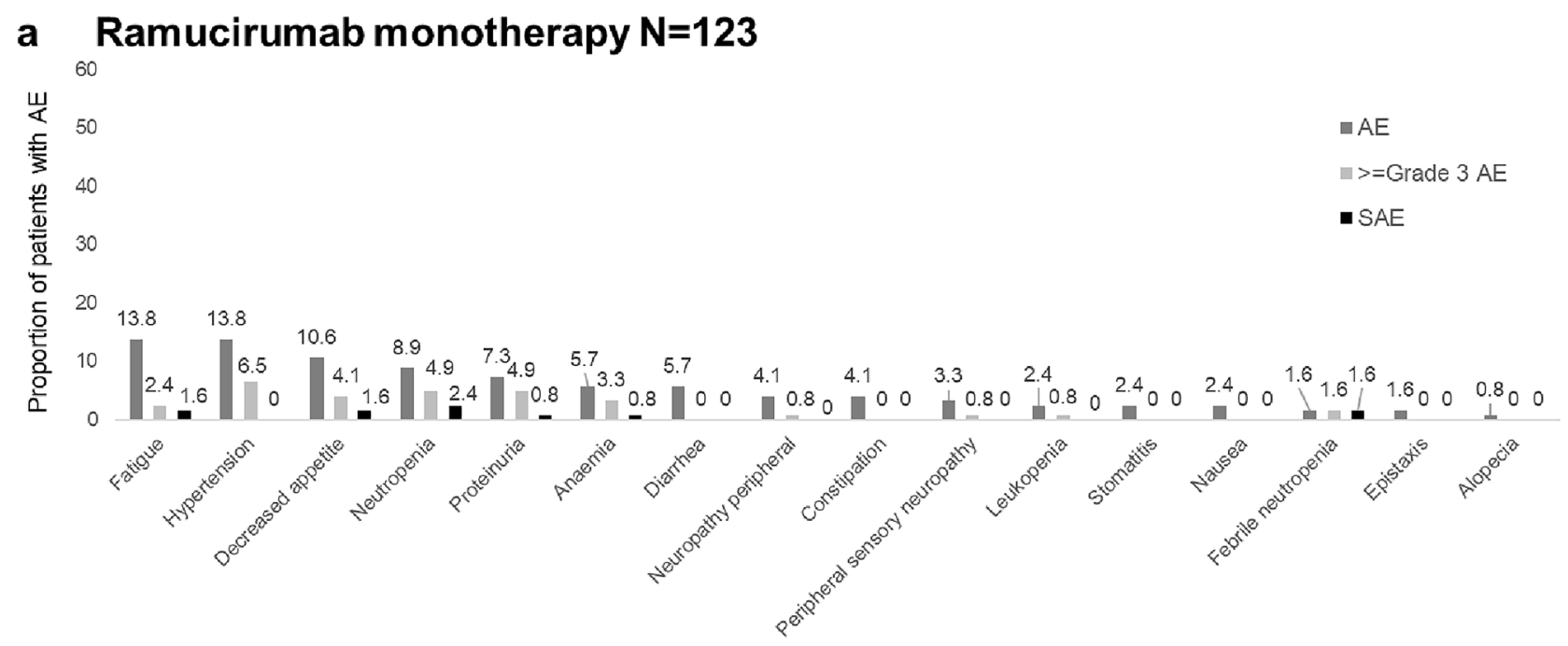

\section{b Ramucirumab + Paclitaxel $\mathrm{N}=528$}

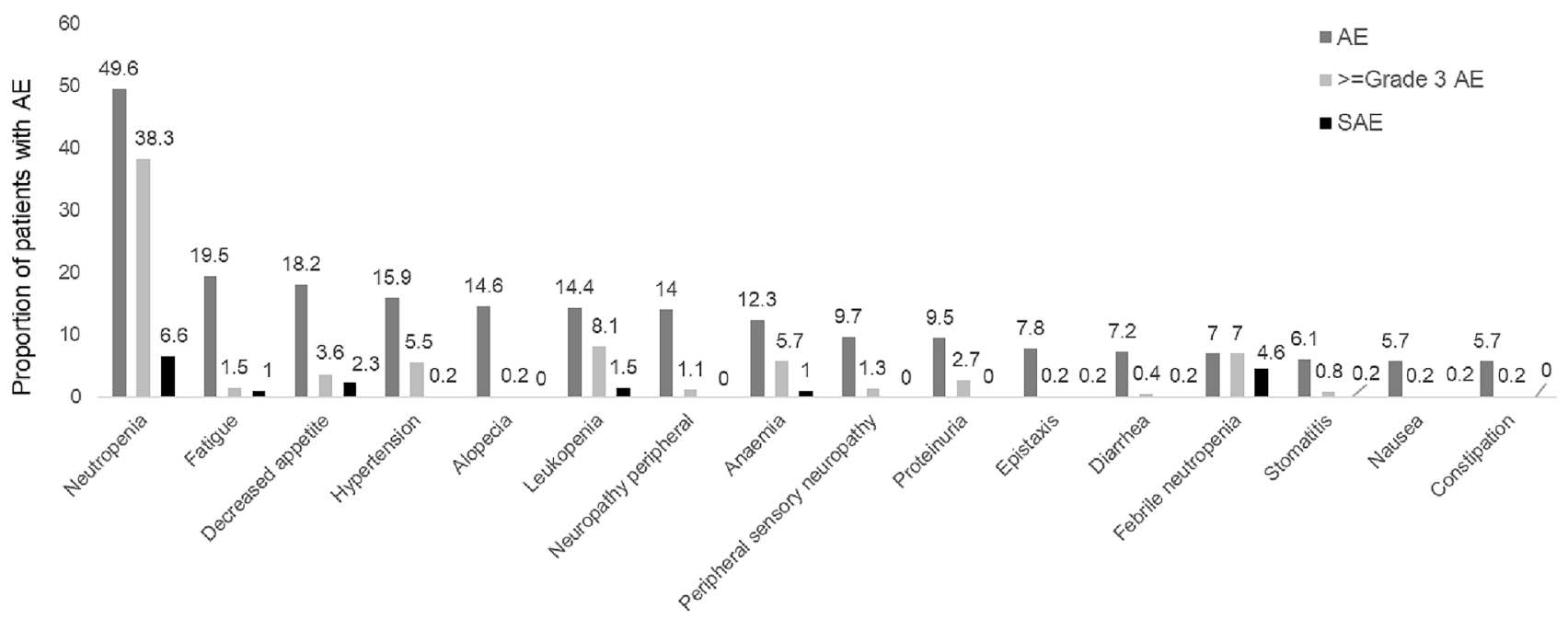

Fig. 1 Adverse events by consolidated terms. AEs reported in $\geq 5 \%$ of patients in either the a ramucirumab monotherapy or $\mathbf{b}$ ramucirumab + paclitaxel combination therapy group are shown. Consolidated terms included the following Medical Dictionary for Regulatory Activities preferred terms (in parentheses) anemia (anemia, hemoglobin decreased); fatigue (fatigue, malaise, asthenia); leukopenia (leukopenia, white blood cell count decreased); neutropenia (neutropenia; neutrophil count decreased), and proteinuria (proteinuria, protein urine, protein urine present). $A E$ adverse event, $S A E$ serious adverse event groups (prior oxaliplatin, 231/268, 86.1\%; no prior oxaliplatin, 329/389; 84.6\%), but the incidence of nervous system disorders was higher in the prior oxaliplatin group (69/268; $25.7 \%$ ) compared with the group without prior oxaliplatin $(73 / 389 ; 18.8 \%)$. The difference in the incidence of nervous system disorders was evident in all grades (grade 1-3) of AEs during the first 4 weeks of ramucirumab treatment but not at later treatment cycles (Fig. 2).

\section{Treatment discontinuation}

Overall, 490 of 658 patients $(74.5 \%)$ had discontinued ramucirumab treatment by 6 months, with 168 patients $(25.5 \%)$ continuing treatment (Table 3). At 12 months, an additional 119 patients had discontinued (accumulated discontinuation 0-12 months: $92.6 \%)$, with $48(7.3 \%)$ still on treatment at the end of the study. A numerically greater proportion of patients in the ramucirumab monotherapy group discontinued compared to the ramucirumab + paclitaxel group (accumulated discontinuation 0-12 months: $98.4 \%$ versus $91.5 \%$, respectively; Table 3 ). The reasons for discontinuation were similar across treatment groups, most commonly progressive disease (75.9\%) and AEs (13.0\%; Table 3). The most common AEs leading to discontinuation were anemia ( $n=12 ; 15.2 \%)$, neutrophil count decreased $(n=10 ; 12.7 \%)$, febrile neutropenia $(n=6 ; 7.6 \%)$, hypertension $(n=6 ; 7.6 \%)$, 
Fig. 2 Incidence of nervous system disorders across cycles stratified by prior oxaliplatin. The proportion of patients reporting nervous system disorders, shown across ramucirumab treatment cycles (weeks) and by the worst grade of adverse event that occurred in each patient

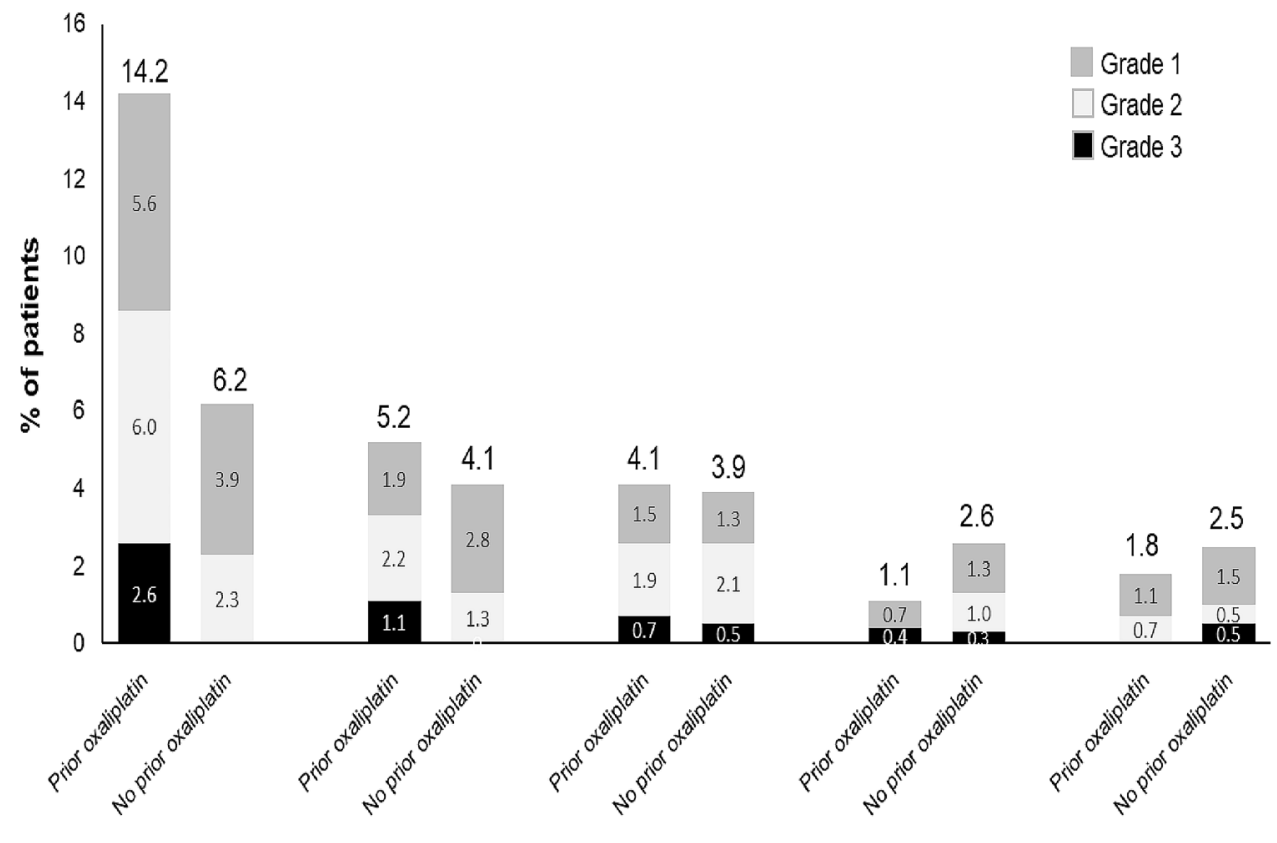

Week $<4$

Week 4 to 8

Week 8 to 12

Week 12 to 16

Week $\geq 16$ and alopecia $(n=5 ; 6.3 \%)$. Five patients discontinued due to neuropathy, all of whom had received prior oxaliplatin $(5 / 268 ; 1.9 \%)$.

\section{Effectiveness}

At the time of database lock, $55.3 \%$ of patients had died in the overall analysis population (364/658 patients), and death was most frequently due to the primary disease (gastric cancer; $98.6 \%$; 359/364 patients). Median survival time was 5.7 months (95\% CI 4.1-6.8 months) in the monotherapy group and 11.0 months (95\% CI 9.8-12.2 months; Fig. 3a and $b$ ) in the ramucirumab + paclitaxel group. Patients with ascites had lower median survival (monotherapy, with ascites: 3.9 months; without ascites: 6.8 months; ramucirumab + paclitaxel, with ascites: 7.3 months; without
Table 3 Discontinuation of ramucirumab

\begin{tabular}{lccc}
\hline & $\begin{array}{l}\text { Ramucirumab mono- } \\
\text { therapy } N=123\end{array}$ & $\begin{array}{l}\text { Ramucirumab + pacli- } \\
\text { taxel } N=528\end{array}$ & $\begin{array}{l}\text { Overall analy- } \\
\text { sis population } \\
N=658^{\mathrm{a}}\end{array}$ \\
\hline $\begin{array}{l}\text { Status at } 6 \text { months } n(\%) \\
\text { Continuing }\end{array}$ & $10(8.1)$ & $154(29.2)$ & $168(25.5)$ \\
Discontinued & $113(91.9)$ & $374(70.8)$ & $490(74.5)$ \\
Status at 12 months $n(\%)$ & $2(1.6)$ & $45(8.5)$ & $48(7.3)$ \\
Continuing & $8(6.5)$ & $109(20.6)$ & $119(18.1)$ \\
Discontinued & $0(0.0)$ & $0(0.0)$ & $1(0.2)$ \\
Not described & & & $462(75.9)$ \\
Reason for discontinuation & $89(73.6)$ & $369(76.4)$ & $79(13.0)$ \\
Progressive disease & $16(13.2)$ & $63(13.0)$ & $28(4.6)$ \\
Adverse event & $7(5.8)$ & $20(4.1)$ & $24(3.9)$ \\
Physician decision & $5(4.1)$ & $19(3.9)$ & $10(1.6)$ \\
Patient decision & $2(1.7)$ & $8(1.7)$ & $3(0.5)$ \\
Death & $1(0.8)$ & $2(0.4)$ & $2(0.3)$ \\
Lost to follow-up & $0(0.0)$ & $2(0.4)$ & $1(0.2)$ \\
Symptom improvement & $1(0.8)$ & $0(0.0)$ & \\
No visits after enrollment & & & \\
\hline
\end{tabular}

$N$ number of patients in population, $n$ number of patients in category

${ }^{a}$ Includes two subgroups with data not shown separately due to small sample size: patients who received other ramucirumab combination therapies $(N=4)$ or unspecified ramucirumab therapy $(N=3)$ 
Ramucirumab monotherapy $\mathrm{N}=123$

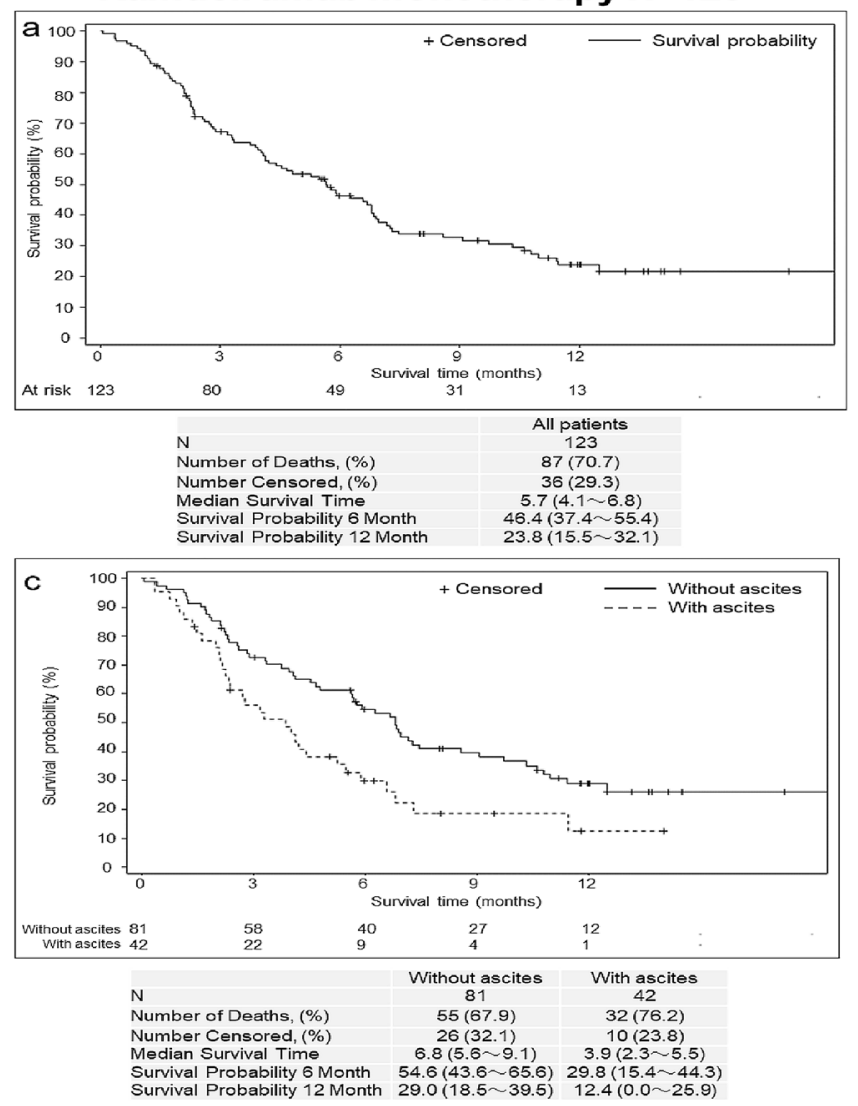

Fig. 3 Survival curves. Kaplan-Meier survival curves shown by treatment category $(\mathbf{a}, \mathbf{b})$ and by the presence and absence of ascites (c, d). Median survival time (months) and 6- and 12-month survival

ascites: 15.5 months; Fig. $3 \mathrm{c}$ and d). Median survival was similar between patients aged $<75$ years and $\geq 75$ years (monotherapy: $<75$ years: 5.3 months; $\geq 75$ years: 6.3 months; ramucirumab + paclitaxel: $<75$ years: 11.2 months; $\geq 75$ years: 10.4 months; Online Resource 6 ).

\section{Discussion}

The current analysis was conducted to provide real-world data on the safety and effectiveness of ramucirumab in patients with advanced gastric cancer in Japan. Our results confirm that the safety profile for ramucirumab in patients under routine clinical care in Japan is in accordance with that observed in prior clinical trials, with no new safety concerns identified. Overall, AEs were reported in $85.3 \%$ of patients, with $55.5 \%$ experiencing $\geq$ Grade 3 AEs and $23.3 \%$ experiencing SAEs. AEs of blood and lymphatic disorders, nervous system disorders, and gastrointestinal disorders were the most frequently reported. The most common SAEs included neutropenia, febrile neutropenia, and
Ramucirumab + Paclitaxel $\mathbf{N}=\mathbf{5 2 8}$

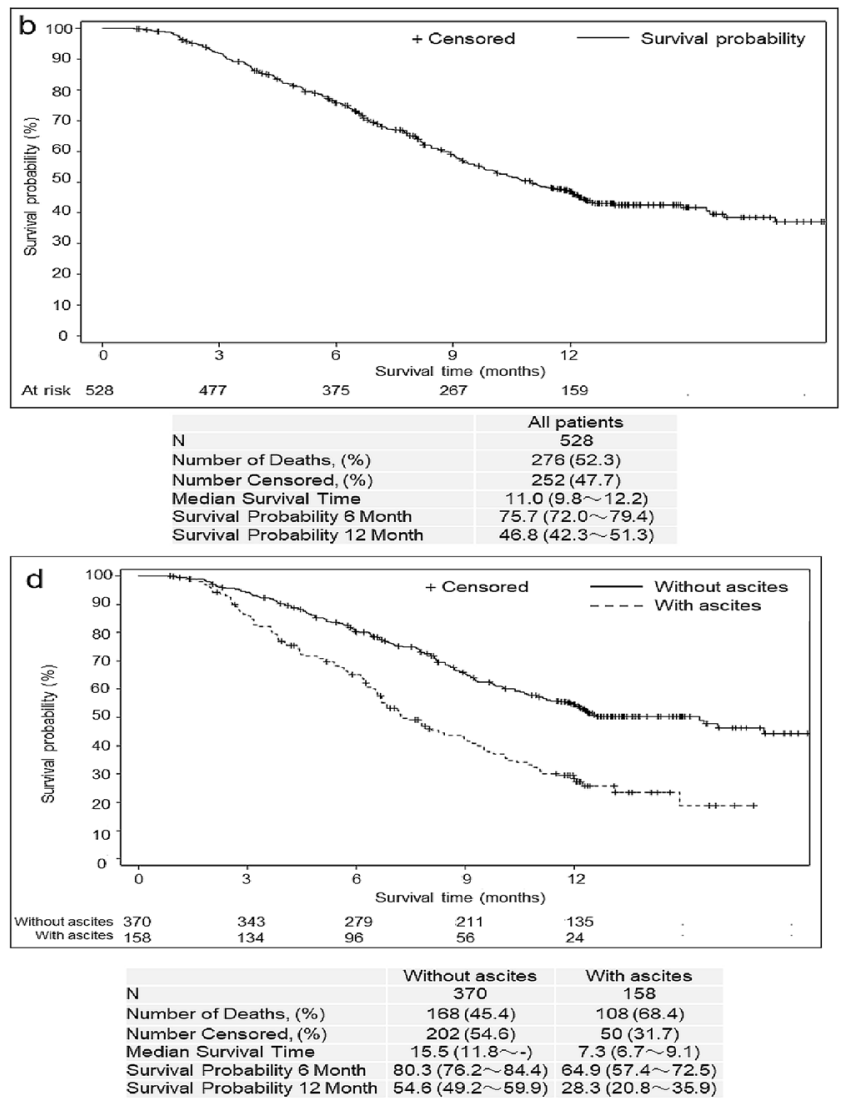

rates (as percentage) are shown with 95\% confidence intervals. - not reached, $N$ number of patients in population

decreased appetite. Hypertension was the most common AESI, reported by $15.7 \%$ of patients, although an SAE of hypertension occurred in only 1 patient. AEs were generally manageable, with most patients discontinuing treatment due to progressive disease (75.9\%) rather than an $\mathrm{AE}$ (13.0\%). AEs in a majority of patients were reported as resolved or resolving at the end of the study period.

Ramucirumab monotherapy was associated with numerically worse baseline ECOG PS and later line of ramucirumab treatment compared with ramucirumab + paclitaxel therapy. The monotherapy group also had a numerically shorter duration of treatment, higher rate of discontinuation, and shorter survival compared with the ramucirumab + paclitaxel therapy group. These differences suggest that patients who received ramucirumab monotherapy may have been in poorer health, and therefore, were not candidates for ramucirumab + paclitaxel combination therapy. AEs were consistent with the known $\mathrm{AE}$ profiles for each drug, with patients in the ramucirumab + paclitaxel group experiencing a numerically greater number of AEs known to be adverse drug reactions for paclitaxel. 
Nearly all patients (98.8\%) had received prior systemic chemotherapy for gastric cancer, which most commonly included tegafur/gimeracil/oteracil potassium and platinumbased drug combinations. Platinum-based drug usage is of particular interest in that peripheral neuropathy and other neurotoxicities are well established, treatment-related AEs associated with oxaliplatin and other platinum-based therapies $[16,17]$. In the current study, the overall incidence of AEs of the nervous system was slightly higher in patients with prior oxaliplatin (25.7\%) compared with those without prior oxaliplatin (18.8\%), with discontinuations due to neuropathy only occurring in the prior oxaliplatin group. Differences between the two groups in the incidence of AEs of neuropathy were most evident during the first 4 weeks of ramucirumab treatment, suggesting that prior oxaliplatin had a limited effect on subsequent ramucirumab treatment in terms of neuropathies.

In the current study, median survival time was 11.0 months for the ramucirumab + paclitaxel group and 5.7 months for the ramucirumab monotherapy group, which demonstrates effectiveness for ramucirumab in line with two prior clinical study studies in global $[5,6]$ and Japanese populations $[18,19]$. Advanced age ( $\geq 75$ years) affected neither survival nor AE incidence rates, indicating that ramucirumab retained effectiveness in elderly patients with gastric cancer without increasing the risk of AEs. In contrast, the presence of ascites was associated with shorter median survival times in both the ramucirumab monotherapy and ramucirumab + paclitaxel groups, which is consistent with prior studies showing poorer prognosis for gastric cancer with malignant ascites [20]. In the phase 3 RAINBOW study, a multivariate analysis using a stepwise Cox model also identified the presence of ascites as a strong independent predictor of survival, with median overall survival times in the placebo group lower in patients with ascites (5.2 months) than in those without ascites (8.5 months) [6, 21]. Nevertheless, ramucirumab + paclitaxel improved survival in patients with gastric cancer both with and without ascites, extending median overall survival by 2.0 and 2.9 months compared with placebo, respectively [21].

The strengths of this study include its prospective design and inclusion of a heterogeneous Japanese population. In addition, the majority of patients in this study received prior tegafur/gimeracil/oteracil potassium, which is commonly used in Japan but not elsewhere, and this increases the value of the current analysis for Japan. However, important limitations inherent to post-marketing surveillance studies should be considered which limit the conclusions that can be drawn, including the lack of a control group, non-interventional design, and low numbers included for AE subgroup analyses. In addition, although separate analyses for ramucirumab monotherapy and combination therapy provided new information on the effectiveness and safety of these regimens in Japanese patients, these results should be interpreted carefully due to the lack of statistical testing of differences and covariate adjustment between subgroups. Furthermore, as the current study had no specific exclusions in the patient population or restrictions on treatment history/concomitant medications, the current data are not directly comparable to the data from prior clinical trials, which had strict sets of eligibility criteria. It is also recognized that $\mathrm{AE}$ incidence is generally lower in post-marketing studies compared with clinical trials, as physicians do not have the same obligations to report AEs in post-marketing studies, which lack the structured data-capturing systems of clinical trials. Nevertheless, with these limitations in mind, it is worth noting that the survival benefit and safety profile in the current real-world setting were broadly similar to the outcomes of prior clinical trials [5, 6, 18, 19]. In Japanese patients who received ramucirumab in the phase 3 RAINBOW study, the incidence of AEs was $100 \%$ and $83.8 \%$ for any grade and $\geq$ Grade 3 AEs, respectively, with neutropenia and lymphopenia the most common AEs reported, and median overall survival was 11.4 months [18].

\section{Conclusion}

This study adds to the limited existing data on ramucirumab in patients with unresectable advanced or recurrent gastric cancer under routine clinical practice, demonstrating that effectiveness and safety outcomes in the real-world setting are consistent with the findings of clinical studies.

Supplementary Information The online version contains supplementary material available at https://doi.org/10.1007/s10120-021-01199-0.

Acknowledgements This work was supported by Eli Lilly Japan K.K., Kobe, Japan. Medical writing assistance (Kaye L. Stenvers, PhD) and editorial assistance (Antonia Baldo, Angela Lorio, and Cynthia Abbott) were provided by Syneos Health and funded by Eli Lilly Japan K.K.

\section{Declarations}

Conflict of interest Yucherng Chen, Taeko Katayose, Soshi Nagaoka, Yongzhe Piao, and Hiroya Asou are employees and minor shareholders of Eli Lilly Japan K.K., Kobe, Japan. Kensei Yamaguchi reports grants and personal fees from Eli Lilly Japan K.K. during the conduct of the study; and grants and personal fees from Taiho Pharmaceutical Co., Ltd, Chugai Pharmaceutical Co., Ltd, Daiichi-Sankyo Co., Ltd, Ono Pharmaceutical Co., Ltd, and Sanofi; grants from Sumitomo Dainippon Pharma Co., Ltd, Boehringer Ingelheim, Gilead Sciences, Inc., and Yakult Honsha Co., Ltd; and personal fees from Merck Serono Co., Ltd, Takeda Pharmaceutical Co., Ltd, Bristol-Myers Squibb, and Bayer, outside the submitted work. 
Ethical approval As a non-interventional post-marketing study, this work was conducted in compliance with the Good Post-marketing Study Practice in Japan. This article does not contain any interventional studies with human or animal subjects performed by any of the authors.

Open Access This article is licensed under a Creative Commons Attribution 4.0 International License, which permits use, sharing, adaptation, distribution and reproduction in any medium or format, as long as you give appropriate credit to the original author(s) and the source, provide a link to the Creative Commons licence, and indicate if changes were made. The images or other third party material in this article are included in the article's Creative Commons licence, unless indicated otherwise in a credit line to the material. If material is not included in the article's Creative Commons licence and your intended use is not permitted by statutory regulation or exceeds the permitted use, you will need to obtain permission directly from the copyright holder. To view a copy of this licence, visit http://creativecommons.org/licenses/by/4.0/.

\section{References}

1. Bray F, Ferlay J, Soerjomataram I, Siegel RL, Torre LA, Jemal A. Global cancer statistics 2018: GLOBOCAN estimates of incidence and mortality worldwide for 36 cancers in 185 countries. CA Cancer J Clin. 2018;68:394-424.

2. Center for Cancer Control and Information Services, National Cancer Center. Projected Cancer Statistics, 2018. https://ganjo ho.jp/en/public/statistics/short_pred.html. Accessed 24 Sep 2020.

3. Muro K, Van Cutsem E, Narita Y, Pentheroudakis G, Baba E, Li J, et al. Pan-Asian adapted ESMO Clinical Practice Guidelines for the management of patients with metastatic gastric cancer: a JSMO-ESMO initiative endorsed by CSCO, KSMO, MOS. SSO TOS Ann Oncol. 2019;30:19-33.

4. Spratlin JL, Cohen RB, Eadens M, Gore L, Camidge DR, Diab S, et al. Phase I pharmacologic and biologic study of ramucirumab (IMC-1121B), a fully human immunoglobulin G1 monoclonal antibody targeting the vascular endothelial growth factor receptor-2. J Clin Oncol. 2010;28:780-7.

5. Fuchs CS, Tomasek J, Yong CJ, Dumitru F, Passalacqua R, Goswami C, et al. Ramucirumab monotherapy for previously treated advanced gastric or gastro-oesophageal junction adenocarcinoma (REGARD): an international, randomised, multicentre, placebocontrolled, phase 3 trial. Lancet. 2014;383:31-9.

6. Wilke H, Muro K, Van Cutsem E, Oh SC, Bodoky G, Shimada Y, et al. Ramucirumab plus paclitaxel versus placebo plus paclitaxel in patients with previously treated advanced gastric or gastrooesophageal junction adenocarcinoma (RAINBOW): a doubleblind, randomised phase 3 trial. Lancet Oncol. 2014;15:1224-35.

7. National Comprehensive Cancer Network, NCCN guidelines. Gastric cancer (Version 2.2020). 2020. https://www.nccn.org/profe ssionals/physician_gls/default.aspx. Accessed 24 Sep 2020.

8. Smyth EC, Verheij M, Allum W, Cunningham D, Cervantes A, Arnold D, ESMO Guidelines Committee. Gastric cancer: ESMO Clinical Practice Guidelines for diagnosis, treatment and followup. Ann Oncol. 2016;27(5):v38-49.
9. Japanese Gastric Cancer Association. Japanese gastric cancer treatment guidelines 2018 (5th edition). Gastric Cancer 2020. https://doi.org/10.1007/s10120-020-01042-y

10. Di Bartolomeo M, Niger M, Tirino G, Petrillo A, Berenato R, Laterza MM, et al. Ramucirumab as second-line therapy in metastatic gastric cancer: real-world data from the RAMoss study. Target Oncol. 2018;13:227-34.

11. Kimura A, Sakai D, Kudo T, Nishida N, Katou A, Inagaki C, et al. The real-world data in patients with advanced gastric cancer treated with ramucirumab combination chemotherapy. J Clin Oncol. 2019;37(4_suppl):156-156.

12. Paulson AS, Hess LM, Liepa AM, Cui ZL, Aguilar KM, Clark J, et al. Ramucirumab for the treatment of patients with gastric or gastroesophageal junction cancer in community oncology practices. Gastric Cancer. 2018;21:831-44.

13. Vogl UM, Vormittag L, Winkler T, Kafka A, Weiser-Jasch O, Heinrich B, et al. Ramucirumab plus paclitaxel or FOLFIRI in platinum-refractory advanced or metastatic gastric or gastroesophageal junction adenocarcinoma-experience at two centres. J Gastrointest Oncol. 2020;11:366-75.

14. Kaplan EL, Meier P. Nonparametric estimation from incomplete observations. J Am Stat Assoc. 1958;53:457-81.

15. Saif MW, Reardon J. Management of oxaliplatin-induced peripheral neuropathy. Ther Clin Risk Manag. 2005;1:249-58.

16. Carozzi VA, Canta A, Chiorazzi A. Chemotherapy-induced peripheral neuropathy: what do we know about mechanisms? Neurosci Lett. 2015;596:90-107.

17. Pulvers JN, Marx G. Factors associated with the development and severity of oxaliplatin-induced peripheral neuropathy: a systematic review. Asia Pac J Clin Oncol. 2017;13:345-55.

18. Shitara K, Muro K, Shimada Y, Hironaka S, Sugimoto N, Komatsu Y, et al. Subgroup analyses of the safety and efficacy of ramucirumab in Japanese and Western patients in RAINBOW: a randomized clinical trial in second-line treatment of gastric cancer. Gastric Cancer. 2016;19:927-38.

19. Yamaguchi K, Fujitani K, Nagashima F, Omuro Y, Machida N, Nishina $T$, et al. Ramucirumab for the treatment of metastatic gastric or gastroesophageal junction adenocarcinoma following disease progression on first-line platinum- or fluoropyrimidinecontaining combination therapy in Japanese patients: a phase 2, open-label study. Gastric Cancer. 2018;21:1041-9.

20. Zheng LN, Wen F, Xu P, Zhang S. Prognostic significance of malignant ascites in gastric cancer patients with peritoneal metastasis: A systemic review and meta-analysis. World J Clin Cases. 2019;7:3247-58.

21. Muro K, Jen MH, Cheng R. Is ramucirumab and paclitaxel therapy beneficial for second-line treatment of metastatic gastric or junctional adenocarcinoma for patients with ascites? Analysis of RAINBOW phase 3 trial data. Cancer Manag Res. 2019;11:2261-7.

Publisher's Note Springer Nature remains neutral with regard to jurisdictional claims in published maps and institutional affiliations. 